TRANSACTIONS OF THE

AMERICAN MATHEMATICAL SOCIETY

Volume 275, Number 2, February 1983

\title{
SKEWNESS IN BANACH SPACES
}

BY

\author{
SIMON FITZPATRICK AND BRUCE REZNICK ${ }^{1}$
}

\begin{abstract}
Let $E$ be a Banach space. One often wants to measure how far $E$ is from being a Hilbert space. In this paper we define the skewness $s(E)$ of a Banach space $E, 0 \leqslant s(E) \leqslant 2$, which describes the asymmetry of the norm. We show that $s(E)=s\left(E^{*}\right)$ for all Banach spaces $E$. Further, $s(E)=0$ if and only if $E$ is a (real) Hilbert space and $s(E)=2$ if and only if $E$ is quadrate, so $s(E)<2$ implies $E$ is reflexive. We discuss the computation of $s\left(L^{p}\right)$ and describe its asymptotic behavior near $p=1,2$ and $\infty$. Finally, we discuss a higher-dimensional generalization of skewness which gives a characterization of smooth Banach spaces.
\end{abstract}

1. Introduction. Let $E$ be a Banach space. One often wants to measure how far $E$ is from being a Hilbert space. In this paper we introduce a new numerical characteristic, skewness, which describes the asymmetry of the norm:

$$
s(E)=\sup \left\{\lim _{t \rightarrow 0^{+}} \frac{\|x+t y\|-\|y+t x\|}{t}: x, y \in E,\|x\|=\|y\|=1\right\} .
$$

It is clear from $(1.1)$ that $0 \leqslant s(E) \leqslant 2$. We show that $s(E)=s\left(E^{*}\right)$ for all Banach spaces $E$. Further, $s(E)=0$ if and only if $E$ is a (real) Hilbert space and $s(E)=2$ if and only if $E$ is quadrate, so $s(E)<2$ implies $E$ is reflexive. We discuss the computation of $s\left(L^{p}\right)$ and describe its asymptotic behavior near $p=1,2$ and $\infty$. Finally, we discuss a higher-dimensional generalization of (1.1) which gives a characterization of smooth Banach spaces.

2. Definitions, notations and preliminaries. For $x, y \in E$ define

$$
\langle x, y\rangle=\|x\| \cdot \lim _{t \rightarrow 0^{+}} \frac{\|x+t y\|-\|x\|}{t},
$$

which is equal to the one-sided derivative of $\frac{1}{2}\|\cdot\|^{2}$ at $x$ in the direction $y$. Let $[x, y]=\langle x, y\rangle-\langle y, x\rangle$; we have the following simple but useful lemma.

LEMMA 2.2. (i) $\langle x, y\rangle=\|x\|\|y\|\langle x /\|x\|, y /\|y\|\rangle$.

(ii) $s(E)=\sup \{[x, y]$ : $\|x\|=\|y\|=1\}=\sup \{[x, y]$ : $\|x\|,\|y\| \leqslant 1\}$.

(iii) $|\langle x, y\rangle| \leqslant\|x\| \cdot\|y\|$.

Proof. (i) This follows from $\langle a x, b y\rangle=a b\langle x, y\rangle$ for $a, b \geqslant 0$.

(ii) The first equality is immediate from (1.1) and (2.1).

Received by the editors November 23, 1981.

1980 Mathematics Subject Classification. Primary 46B20; Secondary 46C05.

'Supported in part by the N.S.F. 
For the second, note that

$$
[x, y]=\|x\|\|y\|\left[\frac{x}{\|x\|}, \frac{y}{\|y\|}\right] .
$$

(iii) This is a consequence of the triangle inequality.

We now determine $s\left(l_{p}\right)$ for $p=1,2$ and $\infty$.

EXAMPLE 2.3. (i) $s\left(l_{2}\right)=0$.

(ii) $s\left(l_{1}\right)=s\left(l_{\infty}\right)=2$.

Proof. (i) If $E=l_{2}$ then $\langle x, y\rangle$ is, in fact, the inner product so that $[x, y] \equiv 0=$ $s\left(l_{2}\right)$.

(ii) For $E=l_{\infty}$, fix $0<\varepsilon<1$ and let $x=(1, \varepsilon-1,0, \ldots)$ and $y=$ $(1-\varepsilon, 1,0, \ldots)$. Then for $t$ sufficiently small, $\|x+t y\|=1+t(1-\varepsilon)$ and $\| y+$ $t x \|=1-t(1-\varepsilon)$. Hence $[x, y]=2(1-\varepsilon)$ and so $s\left(l_{\infty}\right)=2$. Similarly, for $E=l_{1}$ let $x=(\varepsilon, 1-\varepsilon, 0, \ldots)$ and $y=(1-\varepsilon,-\varepsilon, 0, \ldots)$. Thus $[x, y]=2(1-2 \varepsilon)$ and $s\left(l_{1}\right)$ $=2$.

If the norm of $E$ is smooth then $\langle\cdot, \cdot\rangle$ is the "generalized inner product" of Ritt [R]. Ritt showed that a smooth Banach space $E$ satisfying $[x, y] \equiv 0$ is a Hilbert space (see Theorem 3.11 below).

3. The skewness of $E$ and $E^{*}$. In this section we prove those properties of skewness asserted in the introduction. Recall that James $[\mathbf{J}]$ called a space uniformly nonsquare provided there is $\varepsilon>0$ such that, if $\|x\|=\|y\|=1$ and $\|x+y\|>2-\varepsilon$, then $\|x-y\|<2-\varepsilon$. We prefer to follow Day [D] in calling a space quadrate if it fails to be uniformly nonsquare. In the following theorems we exploit, sometimes implicitly, the convexity of the norm function $t \mapsto\|x+t y\|$.

THEOREM 3.1. A Banach space $E$ is quadrate if and only if $s(E)=2$.

Proof. If $s(E)=2$ then for $0<\varepsilon<1$ there exist $x, y \in E$ with $\|x\|=\|y\|=1$ and $[x, y]>2-\varepsilon$. But for $0<t<1$,

$$
\begin{aligned}
& \|x+y\| \geqslant\|x\|+t^{-1}(\|x+t y\|-\|x\|), \\
& \|y-x\| \geqslant\|y\|+t^{-1}(\|y\|-\|y+t x\|) .
\end{aligned}
$$

Upon adding these inequalities and taking the limit as $t \rightarrow 0^{+}$we obtain

$$
\|x+y\|+\|y-x\| \geqslant 2+\lim _{t \rightarrow 0^{+}} \frac{\|x+t y\|-\|y+t x\|}{t}>4-\varepsilon .
$$

Since $\|x \pm y\| \leqslant 2$ we have $\|x \pm y\|>2-\varepsilon$; hence $E$ is quadrate.

Conversely, suppose $E$ is quadrate; for $0<\varepsilon<1$ choose $u, v \in E$ with $\|u\|=$ $\|v\|=1$ and $\|u \pm v\| \geqslant 2-\varepsilon$. Let $w=u+a v$ and $z=v-a u$, where $0<a<1$ will be chosen below. By convexity, for $0<t<a$,

$$
\frac{\|w+t z\|-\|w\|}{t} \geqslant \frac{\|w\|-\|w-a z\|}{a}=\frac{\|w\|-\left\|\left(1+a^{2}\right) u\right\|}{a} .
$$

But $\|w\|=\|u+a v\| \geqslant\|u+v\|-(1-a)\|v\| \geqslant 1+a-\varepsilon$. Therefore,

$$
\frac{\langle w, z\rangle}{\|w\|}=\lim _{t \rightarrow 0^{+}} \frac{\|w+t z\|-\|w\|}{t} \geqslant \frac{(1+a-\varepsilon)-\left(1+a^{2}\right)}{a}=1-a-\frac{\varepsilon}{a} .
$$


Setting $x=w /\|w\|$ and $y=z /\|z\|$, we have by 2.2 (i),

$$
\langle x, y\rangle=\frac{\langle w, z\rangle}{\|w\|\|z\|} \geqslant \frac{1}{\|z\|}\left(1-a-\frac{\varepsilon}{a}\right) \geqslant \frac{1-a-\varepsilon / a}{1+a} .
$$

Similarly,

$$
\frac{\|z\|-\|z+t w\|}{t} \geqslant \frac{\|z\|-\|z+a w\|}{a}=\frac{\|z\|-\left\|\left(1+a^{2}\right) v\right\|}{a}
$$

yields $-\langle z, w\rangle /\|z\| \geqslant 1-a-\varepsilon / a$ and $-\langle y, x\rangle \geqslant(1-a-\varepsilon / a) /(1+a)$. Taking $a=\sqrt{\varepsilon}$ we have $[x, y]=\langle x, y\rangle-\langle y, x\rangle \geqslant(2-4 \sqrt{\varepsilon}) /(1+\sqrt{\varepsilon})$ and, since $\varepsilon$ is arbi$\operatorname{trary}, s(E)=2$.

Corollary 3.2. If $s(E)<2$ then $E$ is reflexive.

Proof. James $[\mathbf{J}]$ showed that a uniformly nonsquare space $E$ (that is, an inquadrate space) must be reflexive. Indeed, $E$ must be super-reflexive (see [D,p. 169]) but we will not use this fact.

To show that $s(E)=s\left(E^{*}\right)$ we introduce the duality map $J: E \rightarrow 2^{E^{*}}$, defined by

$$
J x=\left\{x^{*} \in E^{*}: x^{*}(x)=\|x\|^{2}=\left\|x^{*}\right\|^{2}\right\} .
$$

The following lemma is useful in the sequel. Although it is moderately well-known (see Barbu [B]) we include its short proof for the sake of completeness.

LEMMA 3.3. (i) For $x, y \in E$ there exists $x^{*} \in J x$ such that

$$
x^{*}(y)=\langle x, y\rangle=\lim _{t \rightarrow 0^{+}} \frac{\frac{1}{2}\|x+t y\|^{2}-\frac{1}{2}\|x\|^{2}}{t} .
$$

(ii) If $\|x\|=1, x^{*} \in J x$ and $y \in E$ then $x^{*}(y) \leqslant\|x+y\|-\|x\|$.

Proof. (i) Define a linear functional $g$ on the one-dimensional space $\mathbf{R} y$ by $g(r y)=r\langle x, y\rangle$. Since $g$ is dominated by the sublinear functional $F(z)=\langle x, z\rangle$ we can use the Hahn-Banach theorem to get an element $x^{*}$ of $E^{*}$ with $x^{*}(y)=g(y)$ and $x^{*} \leqslant F$. Then $x^{*}(x) \leqslant F(x)=\|x\|^{2}$ and $-x^{*}(x)=x^{*}(-x) \leqslant-\|x\|^{2}$; hence $x^{*}(x)=\|x\|^{2}$. However, $x^{*}(z) \leqslant F(z)=\langle x, z\rangle \leqslant\|x\|\|z\|$ for all $z \in E$ by Lemma 2.2(iii) so $\left\|x^{*}\right\| \leqslant\|x\|$. Thus $\left\|x^{*}\right\|^{2}=\|x\|^{2}=x^{*}(x)$ and $x^{*} \in J x$.

(ii) Since $x^{*}(x)=1=\left\|x^{*}\right\|$ we have

$$
x^{*}(y)=x^{*}(x+y)-x^{*}(x)=x^{*}(x+y)-\|x\| \leqslant\|x+y\|-\|x\| .
$$

TheOREM 3.4. Let $\bar{s}(E)=\sup \left\{x^{*}(y)-y^{*}(x): x^{*} \in J x, y^{*} \in J y,\|x\|=\|y\|=\right.$ $1\}$; then $\bar{s}(E)=s(E)$.

Proof. Lemmas 2.2(ii) and 3.3(i) together show that $s(E) \leqslant \bar{s}(E)$. To get the reverse inequality, fix $0<\varepsilon<1$ and choose $x, y \in E,\|x\|=\|y\|=1$ and $x^{*} \in J x$, $y^{*} \in J y$ such that $x^{*}(y)-y^{*}(x) \geqslant \bar{s}(E)-\varepsilon$. Thus for $0<t<r<\varepsilon$ we have, by Lemma 3.3(ii) and convexity,

$$
y^{*}(-t x)<\|y-t x\|-\|y\| \leqslant\|y-r x\|-\|y-r x+t x\| .
$$


Dividing by $t$ and letting $t \rightarrow 0^{+}$in (3.5) gives

$$
\begin{aligned}
-y^{*}(x) & \leqslant \lim _{t \rightarrow 0^{+}} \frac{\|y-r x\|-\|y-r x+t x\|}{t} \\
& =-\frac{\langle y-r x, x\rangle}{\|y-r x\|}=-\langle z, x\rangle,
\end{aligned}
$$

where $z=(y-r x) /\|y-r x\|$. But by 3.3(ii) and the triangle inequality,

$$
x^{*}(t y) \leqslant\|x+t y\|-\|x\| \leqslant\|x+t z\|-\|x\|+t\|z-y\| .
$$

Dividing by $t$ in (3.7) and letting $t \rightarrow 0^{+}$gives $x^{*}(y) \leqslant\langle x, z\rangle+\|z-y\|$. In view of (3.6),

$$
x^{*}(y)-y^{*}(x) \leqslant\langle x, z\rangle-\langle z, x\rangle+\|z-y\| .
$$

However, $r<\varepsilon$, so $\|z-y\| \leqslant 2 \varepsilon /(1-\varepsilon)$. From (3.8) we see that $[x, z] \geqslant \bar{s}(E)-\varepsilon$ $-2 \varepsilon /(1-\varepsilon)$, and since $\varepsilon$ is arbitrary and $\|x\|=\|z\|=1$, we conclude that $s(E) \geqslant \bar{s}(E)$.

COROllary 3.9. For any Banach space $E, s(E)=s\left(E^{*}\right)$.

Proof. If $s(E)=2$ then $E$ is quadrate, which implies by [J] that $E^{*}$ is quadrate, so $s\left(E^{*}\right)=2$. If $s(E)<2$ then $E$ is reflexive. By the definitions of $J$ and $\bar{s}(E)$ we may rewrite $\bar{s}(E)$ as

$$
\begin{gathered}
\bar{s}(E)=\sup \left\{x^{*}(y)-y^{*}(x): x, y \in E, x^{*}, y^{*} \in E^{*},\right. \\
\left.x^{*}(x)=y^{*}(y)=\|x\|=\|y\|=\left\|x^{*}\right\|=\left\|y^{*}\right\|=1\right\} .
\end{gathered}
$$

Since (3.10) is symmetric in $E$ and $E^{*}$ and $E^{* *}=E, \bar{s}(E)=\bar{s}\left(E^{*}\right)$. By Theorem 3.4, $s(E)=s\left(E^{*}\right)$.

THEOREM 3.11. If $E$ is a Banach space and $s(E)=0$ then $E$ is a Hilbert space with inner product $\langle\cdot, \cdot\rangle$ and $\langle x, x\rangle=\|x\|^{2}$.

Proof. By Theorem 3.4, $\bar{s}(E)=0$; it follows easily that for all $x, y \in E$ and $x^{*} \in J x, y^{*} \in J y$, we have $x^{*}(y)=y^{*}(x)$. Now pick $x \in E$ and $x^{*} \in J x$. For any $y \in E$ there exists $y^{*} \in J y$ so that $\langle y, x\rangle=y^{*}(x)$ (by 3.3(i)); hence $\langle y, x\rangle=x^{*}(y)$ is linear in $y$ and, similarly, linear in $x$. Finally, $\langle x, x\rangle=\|x\|^{2}$ immediately from the definition. (Alternatively, one can show that $s(E)=0$ implies $E$ is smooth, and apply the main theorem of [R].)

REMARK. Recall that, in Lemma 2.3(ii) and Theorem 3.1, we proved that $s(E)=2$ by exhibiting $x$ and $y$ with $[x, y] \geqslant 2-\varepsilon$. It is natural to ask whether there exist a Banach space $E$ and $x, y \in E$ with $\|x\|=\|y\|=1$ and $[x, y]=2$.

Suppose $[x, y]=2$. Necessarily $\langle x, y\rangle=1$ and by Lemma 3.3, there is $x^{*} \in J x$ with $x^{*}(y)=\langle x, y\rangle=1$, while $x^{*}(x)=1=\left\|x^{*}\right\|^{2}$. Thus $1+t \geqslant\|x+t y\| \geqslant$ $x^{*}(x+t y)=1+t$ for $t \geqslant 0$. Therefore $\|y+t x\|=t\left\|x+t^{-1} y\right\|=t\left(1+t^{-1}\right)=1$ $+t$, so $[x, y]=0$, a contradiction.

4. Skewness of $L^{p}$. We have already seen that $s\left(L^{1}\right)=s\left(L^{\infty}\right)=2, s\left(L^{2}\right)=0$ and $s\left(L^{p}\right)=s\left(L^{q}\right)$ for conjugate $p$ and $q$. In this section, we compute $s(p):=s\left(L^{p}\right)$ for $2<p<\infty$ indirectly (of course, $s(p)=s(p /(p-1)$ ) for $1<p<2$ ), we give 
some particular values and discuss, without detailed proofs, the asymptotics of $s(p)$. Our major result is Theorem 4.1: The proof applies to all $L^{p}(X, \Sigma, \mu)$ provided there are two disjoint elements of $\Sigma$ with positive mass.

TheOREM 4.1. For $2<p<\infty, s(p)=\max _{t>0} 2\left(t-t^{p-1}\right) /\left(1+t^{p}\right)$. This maximum is achieved at $t=t_{p}$, where $t_{p}$ is the unique solution, $0<t_{p}<1$, of $t^{p-1}+t^{1-p}$ $=(p-1)\left(t+t^{-1}\right)$.

Proof. Pick $f, g \in L^{p}$ with $\|f\|=\|g\|=1$. By Lemma 3.3(i), there exist $f^{*} \in J f$ and $g^{*} \in J g$ so that $[f, g]=f^{*}(g)-g^{*}(f)$, but by Hölder's inequality, $h^{*} \in J h$ with $\left\|h^{*}\right\|=\|h\|=1$ implies $h^{*}(\omega)=|h(\omega)|^{p-1} \operatorname{sgn} h(\omega) \mu$-a.e. Accordingly,

$$
\text { . }[f, g]=\int\left(|f|^{p-1}|g|-|f||g|^{p-1}\right) \operatorname{sgn} f \operatorname{sgn} g d \mu \text {. }
$$

(This formula can also be found by differentiating under the integral sign.)

Now suppose that $\lambda$ is chosen so that

$$
\left(|u|^{p-1}|v|-|u||v|^{p-1}\right) \operatorname{sgn} u \operatorname{sgn} v \leqslant \lambda\left(|u|^{p}+|v|^{p}\right)
$$

for all real $u, v$. Then by integrating (4.3) pointwise with $u=f(\omega), v=g(\omega)$, $[f, g] \leqslant 2 \lambda$. In order to determine which $\lambda$ satisfy (4.3) for all real $u, v$, we may assume that $u>0$. If $v>0$ then dividing by $u^{p}$ gives $\lambda \geqslant\left(t-t^{p-1}\right) /\left(1+t^{p}\right)$ for $t=v / u$. If $v<0$, the same inequality arises from dividing by $|v|^{p}$ and letting $t=-u / v$. Accordingly, (4.3) holds when $\lambda=\lambda_{p}=\max \left(t-t^{p-1}\right) /\left(1+t^{p}\right)$. Conversely, let $A$ and $B$ be two sets of positive mass, let $g \equiv t_{p} f$ on $A$ and $f \equiv-t_{p} g$ on $B$, $g>0$ chosen so that $\int_{A} f^{p} d \mu=\int_{B} g^{p} d \mu=\left(t_{p}^{p}+1\right)^{-1}$. Then (4.3) is an equality and $\|f\|=\|g\|=1$ with $[f, g]=2 \lambda_{p}$.

We now present some properties of the function $s(p)$.

THEOREM 4.4. For $2<p<\infty, s(p)$ is monotonically increasing, $s(4)=1 / \sqrt{2}$ and $s(6)=1$.

Proof. Let $w(t, p)=2\left(t-t^{p-1}\right) /\left(1+t^{p}\right)$. Then

$$
\frac{\partial w}{\partial p}=-2 \log t \frac{t^{p-1}+t^{p+1}}{\left(1+t^{p}\right)^{2}}>0
$$

for $0<t<1$. Thus, for $q>p, s(q)=w\left(t_{q}, q\right) \geqslant w\left(t_{p}, q\right)>w\left(t_{p}, p\right)=s(p)$. For $p=4, t_{4}$ is the root of $t^{3}+t^{-3}=3\left(t+t^{-1}\right)$. Letting $u=t_{4}+t_{4}^{-1}, u^{3}-3 u=3 u$, so $u=0, \pm \sqrt{6}$. We thus have $t_{4}+t_{4}^{-1}=\sqrt{6}$, or $t_{4}=(\sqrt{6}-\sqrt{2}) / 2$; it turns out that $w\left(t_{4}, 4\right)=1 / \sqrt{2}$. Similarly, $t_{6}$ is the root of $t^{5}+t^{-5}=5\left(t+t^{-1}\right)$ and, for $u=t_{6}+$ $t_{6}^{-1}, u^{5}-5 u^{3}=0$, so $t_{6}=(\sqrt{5}-1) / 2$ and $w\left(t_{6}, 6\right)=1$.

In general, we cannot hope for an explicit formula for $s(p)$. Indeed the only other tractable value is for $p=3: t_{3}=\left(1+\sqrt{3}-12^{1 / 4}\right) / 2, s(3)=\sqrt{2}(\sqrt{3}-1) 3^{-3 / 4}$. Some numerical computations made on an $H P-41 C$ are shown in Table 4.5. 


$\begin{array}{lclrrr}p & s(p) & p & s(p) & p & s(p) \\ 1 & 2.0000 & 2 & .0000 & 4 & .7071 \\ 1.001 & 1.9829 & 2.001 & .0007 & 5 & .8763 \\ 1.01 & 1.8779 & 2.01 & .0066 & 6 & 1.0000 \\ 1.05 & 1.5803 & 2.1 & .0632 & 7 & 1.0955 \\ 1.1 & 1.3330 & 2.2 & .1207 & 8 & 1.1720 \\ 1.2 & 1.0000 & 2.3 & .1736 & 9 & 1.2349 \\ 1.3 & .7702 & 2.4 & .2224 & 10 & 1.2878 \\ 1.4 & .5951 & 2.5 & .2677 & 20 & 1.5649 \\ 1.5 & .4542 & 2.6 & .3099 & 40 & 1.7430 \\ 1.6 & .3365 & 2.7 & .3493 & 100 & 1.8769 \\ 1.7 & .2357 & 2.8 & .3864 & 1000 & 1.9829 \\ 1.8 & .1477 & 2.9 & .4213 & 10000 & 1.9978 \\ 1.9 & .0698 & 3 & .4542 & \infty & 2\end{array}$

TABLE 4.5

The asymptotic analysis of $s(p)$ is based on the following observations. Suppose $t^{r}+t^{-r}=r\left(t+t^{-1}\right),(r=p-1)$; if $t=e^{z}$ then $\cosh r z=r \cosh z$. Letting $q(u)=$ $u^{-1} \cosh (u)$, we see that $q(r z)=q(z)$. We now look at the equation $q(u)=q(v)$ with $v / u=r$. We state, without proof or error estimates, the following theorem.

THEOREM 4.6. Asymptotically, $s(1+\varepsilon) \simeq 2+2 \varepsilon \log \varepsilon, s(2+\varepsilon) \simeq \alpha|\varepsilon|$ and $s(p)$ $\simeq 2-\alpha \log p / p$ for large $p$. Here, $\alpha=2 y_{0} /\left(y_{0}^{2}-1\right)$, where $y_{0}$ is the root of $(\log y)\left(y^{2}-1\right)=y^{2}+1 ; y_{0} \simeq 3.319, \alpha \simeq .663$.

5. Generalizations of skewness. In this section we generalize skewness to allow for an arbitrary (finite) number of points and derive a family of characterizations of smooth Banach spaces. We wish to thank Professor A. Pelczynski for suggesting to us a generalization of this kind.

For $n \geqslant 2$ and $x_{i} \in E$, define the following expression:

$$
\left[x_{1}, \ldots, x_{n}\right]_{n}=\limsup _{\underline{t} \rightarrow \underline{0}} \frac{\sum \operatorname{sgn}(\sigma)\left\|x_{\sigma(1)}+t_{2} x_{\sigma(2)}+\cdots+t_{n} x_{\sigma(n)}\right\|}{\left|t_{2}\right|+\cdots+\left|t_{n}\right|}
$$

where the summation in (5.1) is taken over all permutations $\sigma \in S_{n}$. Note that for $n=2$ we have $[x, y]_{2}=\max ([x, y],[x,-y])$. We now define $n$-dimensional skewness:

$$
s_{n}(E)=\sup \left\{\left[x_{1}, \ldots, x_{n}\right]_{n}:\left\|x_{1}\right\|=\cdots=\left\|x_{n}\right\|=1\right\} ;
$$

observe that $s_{2}(E)=s(E)$. Since $n$ is clear from the context, we drop the outer subscript on $\left[x_{1}, \ldots, x_{n}\right]$ for $n \geqslant 3$. We will establish a peculiar branching in the behavior of $s_{\mathrm{n}}: s_{3}$ is much like $s_{2}$, but $s_{n}$ for $n \geqslant 4$ selects out nonsmoothness in Banach spaces.

THEOREM 5.3. If $E$ is a smooth Banach space then $2 s(E) \leqslant s_{3}(E) \leqslant 3 s(E)$. 
Proof. Letting $u^{*}$ denote the derivative of the norm at $u$, a routine computation shows that

$$
[x, y, z]=\limsup _{\left(t_{2}, t_{3}\right) \rightarrow(0,0)}\left\{\frac{t_{2}-t_{3}}{\left|t_{2}\right|+\left|t_{3}\right|}\left(x^{*}(y-z)+y^{*}(z-x)+z^{*}(x-y)\right)\right\} .
$$

By Theorem 3.4, $x^{*}(y)-y^{*}(x) \leqslant s(E)$, etc., so $[x, y, z] \leqslant 3 s(E)$. On the other hand, pick $z=-y$ and take $t_{2}>0, t_{3}=0$ in (5.4). Since $(-y)^{*}=-y^{*},[x, y,-y] \geqslant$ $x^{*}(2 y)-2 y^{*}(x)=2\left(x^{*}(y)-y^{*}(x)\right)$. If we now take the supremum over $\|x\|=$ $\|y\|=1$, we get $s_{3}(E) \geqslant 2 s(E)$.

A more delicate analysis is necessary when $E$ is not smooth.

LEMMA 5.5. Suppose $x, y \in E$ and $\alpha, \beta$ are real, with $\|x\|=1$. Then

$$
\|(1+\alpha t) x+\beta t y\|=\|x+\beta t y\|+\alpha t+o(t)
$$

for small $t$.

Proof. We have $\|(1+\alpha t) x+\beta t y\|=(1+\alpha t)\left\|x+\beta t(1+\alpha t)^{-1} y\right\|=$ $(1+\alpha t)(\|x+\beta t y\|+o(t))=\|x+\beta t y\|+\alpha t\|x\|+o(t)$.

THEOREM 5.6. For any Banach space $E, s_{3}(E) \geqslant 2 s(E)$.

Proof. As in the proof of Theorem 5.3, we compute $[u, v,-v]$ for appropriate $u, v \in E$. Taking $t_{2}=t>0$ and $t_{3}=0$ in (5.1), we have, after a rearrangement of terms,

$$
[u, v,-v] \geqslant \lim _{t \rightarrow 0^{+}}\left(\frac{\|u+t v\|-\|v+t u\|}{t}+\frac{\|u-t v\|-\|v-t u\|}{-t}\right) .
$$

Thus it suffices to find $u, v$ so that $\|u\|=\|v\|=1$ and

$$
\liminf _{t \rightarrow 0} \frac{\|u+t v\|-\|v+t u\|}{t} \geqslant s(E)-2 \varepsilon
$$

Pick $x, y$ with $\|x\|=\|y\|=1$ and $[x, y] \geqslant s(E)-\varepsilon$. For any $\alpha>0$, the convexity of the norm implies

$$
\begin{gathered}
\liminf _{t \rightarrow 0} \frac{\|x+\alpha y+t y\|-\|x+\alpha y\|}{t} \geqslant\langle x, y\rangle, \\
\limsup _{t \rightarrow 0} \frac{\|y-\alpha x+t y\|-\|y-\alpha x\|}{t} \leqslant\langle y, x\rangle .
\end{gathered}
$$

Let $u=(x+\alpha y) /\|x+\alpha y\|$ and $v=(y-\alpha x) /\|y-\alpha x\|$, where $0<\alpha<\varepsilon / 5$, so $\|u-x\|,\|v-y\| \leqslant 2 \alpha /(1-\alpha)<\varepsilon / 2$. Then

$$
\text { (5.10) } \begin{aligned}
\liminf _{t \rightarrow 0} \frac{\|u+t v\|-1}{t} & =\liminf _{t \rightarrow 0} \frac{\|x+\alpha y+t\| x+\alpha y\|y\|-\|x+\alpha y\|}{t\|x+\alpha y\|} \\
& =\liminf _{t \rightarrow 0} \frac{\|x+\alpha y+t y\|-\|x+\alpha y\|}{t} \geqslant\langle x, y\rangle .
\end{aligned}
$$


Similarly, $\lim \sup _{t \rightarrow 0} t^{-1}(\|v+t x\|-1) \leqslant\langle y, x\rangle$ so that

$$
\liminf _{t \rightarrow 0} \frac{\|u+t y\|-\|v+t x\|}{t} \geqslant\langle x, y\rangle-\langle y, x\rangle \geqslant s(E)-\varepsilon .
$$

Since $\|u-x\|,\|v-y\| \leqslant \varepsilon / 2$, (5.11) implies (5.8) and we are done.

Corollary 5.12. If $s_{3}(E)=0$ then $E$ is a Hilbert space.

THEOREM 5.13. For any Banach space $E, s_{3}(E) \leqslant 3 s(E)$.

Proof. For $x, y \in E$, let $z=x /\|x\|$. Then by Lemma 3.3(ii), $\|x\|-\|x+y\|=$ $\|x\|(\|z\|-\|z+y /\| x\|\|) \leqslant-\|x\| z^{*}(y /\|x\|)=-z^{*}(y)=z^{*}(-y)$ for $z^{*} \in J z$. Thus, for any $u, v, w \in E$,

$$
\left\|u+t_{2} v+t_{3} w\right\|-\left\|u+t_{2} w+t_{3} v\right\| \leqslant u^{*}\left(\left(t_{2}-t_{3}\right)(v-w)\right)
$$

for $u^{*} \in J\left(\left(u+t_{2} v+t_{3} w\right) /\left\|u+t_{2} v+t_{3} w\right\|\right)$. For $(u, v, w)=\left(x_{1}, x_{2}, x_{3}\right)$, $\left(x_{2}, x_{3}, x_{1}\right),\left(x_{3}, x_{1}, x_{2}\right)$, let $u^{*}$ be denoted by $x_{1}^{*}, x_{2}^{*}, x_{3}^{*}$ respectively. These functionals depend on $t_{2}$ and $t_{3}$ of course. Then

$$
\left[x_{1}, x_{2}, x_{3}\right] \leqslant \limsup _{\underline{t} \rightarrow \underline{0}} \frac{t_{2}-t_{3}}{\left|t_{2}\right|+\left|t_{3}\right|}\left\{x_{1}^{*}\left(x_{2}-x_{3}\right)+x_{2}^{*}\left(x_{3}-x_{1}\right)+x_{3}^{*}\left(x_{1}-x_{2}\right)\right\} \text {. }
$$

As $\left(t_{2}, t_{3}\right) \rightarrow(0,0)$ we can choose weak* cluster points $y_{i}^{*}$ for $x_{i}^{*}$ since the unit ball is weak* compact in $E^{*}$. It is easily checked that $y_{i}^{*} \in J\left(x_{i}\right)$ and, since $t_{2}-t_{3} \leqslant\left|t_{2}\right|$ $+\left|t_{3}\right|,\left[x_{1}, x_{2}, x_{3}\right] \leqslant y_{1}^{*}\left(x_{2}-x_{3}\right)+y_{2}^{*}\left(x_{3}-x_{1}\right)+y_{3}^{*}\left(x_{1}-x_{2}\right)$. As in the proof of Theorem 5.3 this implies $s(E) \leqslant 3 s(E)$.

When $n \geqslant 4$ there is again a dichotomy between smooth and nonsmooth Banach spaces. The differences, however, are now irreconcilable.

THEOREM 5.16. For some $i, 1 \leqslant i \leqslant n$, let $x_{i}$ be a point of smoothness for the norm of E and suppose $n \geqslant 4$. Then

$$
\limsup _{\underline{t} \rightarrow \underline{0}} \frac{\sum ' \operatorname{sgn}(\sigma)\left\|x_{\sigma(1)}+t_{2} x_{\sigma(2)}+\cdots+t_{n} x_{\sigma(n)}\right\|}{\left|t_{2}\right|+\cdots+\left|t_{n}\right|}=0
$$

where the summation in (5.17) is taken over all $\sigma \in S_{n}$ with $\sigma(1)=i$.

Proof. Let $x_{i}^{*}$ denote the derivative of the norm at $x_{i}$; then we can rewrite (5.17) as (5.18)

$$
\limsup _{\underline{t} \rightarrow \underline{0}} \frac{\sum \operatorname{sgn}(\sigma)\left(\left\|x_{i}\right\|+\sum_{j=2}^{n} t_{j} x_{i}^{*}\left(x_{\sigma(j)}\right)+o(t)\right)}{\left|t_{2}\right|+\cdots+\left|t_{n}\right|},
$$

or, reversing the order of summation,

$$
\limsup _{\underline{t} \underline{0} \underline{j=2}} \frac{\sum_{j=1}^{n} \sum_{k=1}^{n}\left(\sum_{j, k} \operatorname{sgn}(\sigma)\right)\left(t_{j} x_{i}^{*}\left(x_{k}\right)+\left\|x_{i}\right\|\right)}{\left|t_{2}\right|+\cdots+\left|t_{n}\right|},
$$


where $\Sigma_{j, k}^{\prime}$ denotes the sum over all permutations $\sigma$ for which $\sigma(1)=i, \sigma(j)=k$. Since $n \geqslant 4$, an equal number of such permutations are even and odd; thus the inner sum vanishes and the theorem is proved.

Corollary 5.19. For any smooth Banach space $E, s_{n}(E)=0$ for $n \geqslant 4$.

When $n \geqslant 4$, smoothness is also necessary for $s_{n}(E)=0$, as we now show.

THEOREM 5.20. If $E$ is not smooth then $s_{n}(E)>0$ for every $n$.

Proof. If $n=2,3$, only Hilbert spaces have $s_{n}(E)=0$. Let $n \geqslant 4$ and let $x$ be a point of nonsmoothness in the direction $y,\|x\|=\|y\|=1$ and, without loss of generality, suppose that $E$ is spanned by $x$ and $y$ (this can only decrease $s_{n}(E)$ ). Since $E$ is two-dimensional, it follows from (5.5) that the norm is not smooth at $x$ in any direction of $E$ other than $\pm x$. On the other hand, the function $t \mapsto\|x+t y\|$ is convex and so is differentiable except at a countable number of points. By considering $y^{\prime}=r x+s y,\left\|y^{\prime}\right\|=1$ if necessary, we can assume that the norm is smooth at $y$; further, we can select a large number of elements $\alpha x+\beta y,\|\alpha x+\beta y\|=1$, at which the norm is smooth.

Let $d_{+}$and $d_{-}$denote $\lim _{t \rightarrow 0^{ \pm}} t^{-1}(\|x+t y\|-\|x\|)$; without loss of generality, suppose $d_{+}>d_{-}$. We now let $x_{1}=x, x_{2}=-y$ and $x_{i}=\alpha_{i} x+\beta_{i} y$ for $3 \leqslant i \leqslant n$; where $\left\|x_{i}\right\|=1$, the norm is smooth at $x_{i}, i \geqslant 2$, and $(n-2)^{-1}>\beta_{3}>\cdots>\beta_{n}>0$. Choose $\lambda_{2}, \ldots, \lambda_{n}$ with $\lambda_{2}=1$ and $\lambda_{3}>\cdots>\lambda_{n}>1$ so that $\sum_{i=3}^{n} \beta_{i} \lambda_{i}>1>$ $\sum_{i=3}^{n} \beta_{i} \lambda_{\pi(i)}$ for any permutation $\pi$ of $\{2, \ldots, n\}$ other than the identity. Consider (5.1) with $t_{i}=\lambda_{i} t, t>0$, and let $\lambda=\sum_{i=2}^{n} \lambda_{i}$. We have

$$
\lambda\left[x_{1}, \ldots, x_{n}\right] \geqslant \lim _{t \rightarrow 0^{+}} t^{-1} \sum{ }^{\prime} \operatorname{sgn}(\sigma)\left\|x+t x_{\sigma(2)}+\lambda_{3} t x_{\sigma(3)}+\cdots+\lambda_{n} t x_{\sigma(n)}\right\|,
$$

where $\Sigma^{\prime}$ denotes the sum over permutations with $\sigma(1)=1$; the contribution from the other $\sigma$ 's vanishes by Theorem 5.16. We might as well sum in (5.21) over $\sigma^{-1}$; hence,

$$
\begin{aligned}
\lambda\left[x_{1}, \ldots, x_{n}\right] & \geqslant \lim _{t \rightarrow 0^{+}} t^{-1} \sum ' \operatorname{sgn}(\sigma)\left\|x+t \lambda_{\sigma(2)}(-y)+\sum_{i=3}^{n} t \lambda_{\sigma(i)}\left(\alpha_{i} x+\beta_{i} y\right)\right\| \\
& =\lim _{t \rightarrow 0^{+}} t^{-1} \sum \operatorname{sgn}(\sigma)\left\|\left(1+\sum_{i=3}^{n} t \lambda_{\sigma(i)} \alpha_{i}\right) x+\left(\sum_{i=3}^{n} \beta_{i} \lambda_{\sigma(i)}-\lambda_{\sigma(2)}\right) t y\right\| .
\end{aligned}
$$

Apply Lemma 5.5 to (5.22) and note that $\Sigma^{\prime} \operatorname{sgn}(\sigma) \sum_{i=3}^{n} \lambda_{\sigma(i)} \alpha_{i}$ vanishes; thus,

$$
\lambda\left[x_{1}, \ldots, x_{n}\right] \geqslant \lim _{t \rightarrow 0^{+}} t^{-1} \sum{ }^{\prime} \operatorname{sgn}(\sigma)\left\|x+\left(\sum_{i=3}^{n} \beta_{i} \lambda_{\sigma(i)}-\lambda_{\sigma(2)}\right) t y\right\| .
$$


By choice, $\sum_{i=3}^{n} \beta_{i} \lambda_{i}-1$ is positive, but for other $\sigma$ 's, $\sum_{i=3}^{n} \beta_{i} \lambda_{\sigma(i)}-\lambda_{\sigma(2)}$ is negative. Recalling the definition of $d_{ \pm}$, we have

$$
\begin{aligned}
\lambda\left[x_{1}, \ldots, x_{n}\right] & =\left(\sum_{i=3}^{n} \beta_{i} \lambda_{i}-1\right) d_{+}+\left(\sum_{\sigma \neq \mathrm{id}} \operatorname{sgn}(\sigma)\left(\sum_{i=3}^{n} \beta_{i} \lambda_{\sigma(i)}-\lambda_{\sigma(2)}\right)\right) d_{-} \\
& =\left(\sum_{i=3}^{n} \beta_{i} \lambda_{i}-1\right)\left(d_{+}-d_{-}\right)>0 .
\end{aligned}
$$

We conclude with some computations.

THEOREM 5.25. (i) For $1<p<\infty$ and $p^{-1}+q^{-1}=1, s_{3}\left(L^{p}\right)=s_{3}\left(L^{q}\right)$.

(ii) For $1<p<\infty, s_{3}\left(L_{p}\right)=3 \sup \Phi(r, s, t)\left(|r|^{p}+|s|^{p}+|t|^{p}\right)^{-1}$, where

$$
\begin{aligned}
\Phi(r, s, t)= & \left(|r|^{p-1}|s|-|r||s|^{p-1}\right) \operatorname{sgn}(r s)+\left(|s|^{p-1}|t|-|s||t|^{p-1}\right) \operatorname{sgn}(s t) \\
& +\left(|t|^{p-1}|r|-|t||r|^{p-1}\right) \operatorname{sgn}(r t) .
\end{aligned}
$$

Proof. (i) Since $L^{p}$ is smooth, by (5.4) and the proof of Theorem 4.1, $[f, g, h]=$ $\int \Phi(f, g, h) d \mu$. For $k \in L^{p}$, let $k^{*}(\omega)=|k(\omega)|^{p-1} \operatorname{sgn} k(\omega)$. Then $k^{*} \in$ $L^{q}(p-1=p / q),\left\|k^{*}\right\|=1$ and it is easily checked that $[f, g, h]=\left[f^{*}, h^{*}, g^{*}\right]$ so that $s_{3}\left(L^{p}\right) \leqslant s_{3}\left(L^{q}\right) \leqslant s_{3}\left(L^{p}\right)$.

(ii) Let $\lambda_{p}$ denote the supremum of $\Phi(r, s, t)\left(|r|^{p}+|s|^{p}+|t|^{p}\right)^{-1}$. Then, as in the proof of Theorem 4.1, $s_{3}\left(L^{p}\right) \leqslant 3 \lambda_{p}$ and the maximum is achieved since we may assume it to occur on $|r|^{p}+|s|^{p}+|t|^{p}=1$. Provided $(X, \mu)$ has three disjoint sets of positive mass, we can construct $f, g, h$ for which $[f, g, h]=3 \lambda_{p}$, as before.

We remark that it seems very hard to compute $s_{3}\left(L^{p}\right)$. The only nontrivial explicit value we know is $s_{3}\left(L_{4}\right)=(3 / 2)^{7 / 4} \simeq 2.0331$. The computation is very indirect, following from representation of

$$
r^{4}+s^{4}+t^{4}-2^{7 / 4} 3^{-3 / 4}\left(r^{3}(s-t)+s^{3}(t-r)+t^{3}(r-s)\right)
$$

as a sum of squares of quadratic forms.

EXAMPLE 5.26. (i) $s_{3}\left(l_{1}\right)=s_{3}\left(l_{\infty}\right)=6$.

(ii) $s_{4}\left(l_{\infty}\right) \geqslant 6$.

Proof. (i) For any $E, s_{3}(E) \leqslant 3 s(E) \leqslant 6$. On the other hand, take $\underline{t}=(t, 0)$ and points $(1-2 \varepsilon, \varepsilon,-\varepsilon),(-\varepsilon, 1-2 \varepsilon, \varepsilon),(\varepsilon,-\varepsilon, 1-2 \varepsilon)$ for $l_{1}$ and $(1, \varepsilon-1,1-\varepsilon)$, $(1-\varepsilon, 1, \varepsilon-1),(\varepsilon-1,1-\varepsilon, 1)$ for $l_{\infty}$, where $\varepsilon>0$ is small. The calculations resemble (2.3).

(ii) Take $\underline{t}=(t, 0)$ and $x_{i} \in l_{\infty}^{12}$, where $x_{1}=(A, B, C, D), x_{2}=(B, A, D, C)$, $x_{3}=(C, D, A, B), x_{4}=(D, C, B, A), A=(1,1,1), B=(\alpha, 0,-\alpha), C=(-\alpha, \alpha, 0)$, $D=(0,-\alpha, \alpha)$ and $\alpha=1-\varepsilon$.

Questions 5.27. Is $s_{3}(E)=s_{3}\left(E^{*}\right)$ ? (There are well-known smooth $E$ with nonsmooth $E^{*}$ and vice versa.) What are $s_{n}\left(l_{1}\right), s_{n}\left(l_{\infty}\right)$ for $n \geqslant 4$ ? What is the behavior of $n \mapsto s_{n}(E)$ for $n \geqslant 2$ ? What is $\sup _{E} s_{n}(E)$ ? For which spaces is it achieved?

We wish to thank the referee for several helpful suggestions. 


\section{REFERENCES}

[B] Viorel Barbu, Nonlinear semigroups and differential equations in Banach spaces, Noordhoff, Leyden, 1976.

[D] Mahlon M. Day, Normed linear spaces, 3rd ed., Springer-Verlag, New York, 1973.

[J] R. C. James, Uniformly non-square Banach spaces, Ann. of Math. (2) 80 (1964), 542-550.

[R] R. K. Ritt, A generalization of inner product, Michigan Math. J. 3 (1955), 23-26.

Department of Mathematics, University of Illinois at Urbana-Champaign, Urbana, Illinois 61801 (Current address of Bruce Reznick)

Current address (Simon Fitzpatrick): Department of Mathematics, University of Auckland, Private Bag, Auckland, New Zealand 